\title{
Space and time in perceptual causality
}

\author{
Benjamin Straube ${ }^{1,2 *}$ and Anjan Chatterjee ${ }^{1}$ \\ 1 Department of Neurology and the Center for Cognitive Neuroscience, University of Pennsylvania, Philadelphia, PA, USA \\ 2 Department of Psychiatry and Psychotherapy, Philipps-University Marburg, Marburg, Germany
}

\author{
Edited by: \\ Anna C. Nobre, University of Oxford, \\ UK \\ Reviewed by: \\ Jill O'Reilly, University of Oxford, UK \\ Jennifer T. Coull, Universite Aix- \\ Marseille 1, France \\ *Correspondence: \\ Benjamin Straube, Department of \\ Psychiatry and Psychotherapy, Philipps- \\ University Marburg, Rudolf-Bultmann- \\ Straße 8, D-35039 Marburg, Germany. \\ e-mail: straubeb@med.uni-marburg.de
}

Inferring causality is a fundamental feature of human cognition that allows us to theorize about and predict future states of the world. Michotte suggested that humans automatically perceive causality based on certain perceptual features of events. However, individual differences in judgments of perceptual causality cast doubt on Michotte's view. To gain insights in the neural basis of individual difference in the perception of causality, our participants judged causal relationships in animations of a blue ball colliding with a red ball (a launching event) while fMRIdata were acquired. Spatial continuity and temporal contiguity were varied parametrically in these stimuli. We did not find consistent brain activation differences between trials judged as caused and those judged as non-caused, making it unlikely that humans have universal instantiation of perceptual causality in the brain. However, participants were slower to respond to and showed greater neural activity for violations of causality, suggesting that humans are biased to expect causal relationships when moving objects appear to interact. Our participants demonstrated considerable individual differences in their sensitivity to spatial and temporal characteristics in perceiving causality. These qualitative differences in sensitivity to time or space in perceiving causality were instantiated in individual differences in activation of the left basal ganglia or right parietal lobe, respectively. Thus, the perception that the movement of one object causes the movement of another is triggered by elemental spatial and temporal sensitivities, which themselves are instantiated in specific distinct neural networks.

Keywords: perception of causality, fMRI, spatial continuity, temporal contiguity, expectation

\section{INTRODUCTION}

Inferring cause and effect is central to our understanding of the physical world. This ability is critical in predicting future states and may be a fundamental feature that differentiates humans from animals and primates. For example, Wolpert suggests that causal thinking underlies the development of complex tools and subsequent advantages in evolutional natural selection followed by a host of unique human achievements (see Wolpert, 2006 or Wolpert, 2009). Philosophers and scientists have grappled with how we know that one event causes another, even in simple events such as one billiard ball causing another to move (Hume, 1740/1960, 1748/1977). Hume argued that our concept of causality must arise from repeated experiences and associated relationships. He believed that sensory events could not give rise directly to the experience of causality, and rejected the possibility of causal perception.

In contrast with Hume, Michotte (1946/1963) in the mid twentieth century, suggested the existence of something like a "causal impression" (p. 255). Remarkably, his simple stimuli investigating the perception of physical causality corresponded closely to Hume's favorite example of one billiard ball hitting another. Michotte named this family of events, in which Object A approaches and contacts Object B, followed immediately by movement of Object B, "launching events". He demonstrated that these simple stimuli are sufficient to produce the perception of causality. Based on more than 100 observations, Michotte claimed that causality is perceived directly, a view that has since accumulated considerable support (e.g., Leslie, 1982; Leslie and Keeble, 1987; Oakes and Cohen, 1990; Scholl and Tremoulet, 2000; Blakemore et al., 2001).

Two observations raise doubts about the hypothesis that causality is perceived directly and automatically. First, prolonged exposure to obviously causal or non-causal events changes perceptions of causality (Gruber et al., 1957; Powesland, 1959). Second, there appear to be individual differences in judgments of causality (Gemelli and Cappellini, 1958; Beasley, 1968). These findings raise the possibility that the perception of causality might be learned rather than apprehended automatically. Several parameters contribute to the perception of causality (see Michotte, 1946/1963). Spatial continuity and temporal contiguity have received most attention in behavioral, and cognitive neuroscience studies (see Scholl and Tremoulet, 2000; Blakemore et al., 2003; Fugelsang et al., 2005; Roser et al., 2005; Schlottmann et al., 2006). Whereas the effect of time delays on the judgment of causality has often been the subject of previous research (e.g., Schlottmann and Shanks, 1992; Schlottmann and Anderson, 1993; Guski and Troje, 2003; Schlottmann et al., 2006), the effect of movement direction has been less studied. Similar to the effect of increasing time delays (where small delays of 100-200 ms may minimize the perception of causality; see for example Schlottmann and Anderson, 1993), Michotte reported a parametric decline with increase in changes in movement direction, with causal perception dissipating at $90^{\circ}$ angles. However, Beasley (1968) investigated 181 participants and found that up to $45 \%$ of participants still described events with $90^{\circ}$ angle changes as causal. 
In previous research, most spatial violations were defined as physical gaps between stimuli (the objects never make contact) (e.g., Fugelsang et al., 2005; Roser et al., 2005; Schlottmann et al., 2006). The advantage of inducing spatial violations by variation of movement direction is that contact between the objects occurs in every event. To our knowledge, no study has investigated the brain processes underlying the effect of variation of the movement direction between two objects on the judgment of causality.

Recent $\mathrm{fMRI}$ studies have focused on increases in activation during conditions defined as causal than in those defined as non-causal (Blakemore et al., 2001; Fonlupt, 2003; Fugelsang et al., 2005). This categorization was performed on, for example, extreme violations of temporal and spatial parameters and was further validated in separate behavioral experiments (Fugelsang et al., 2005). However, this procedure is insensitive to detect neural processes related to individual differences in sensitivity to perceptual cues used for the judgment of causality.

Individual differences are present when small violations of spatial continuity or temporal contiguity are introduced (Schlottmann and Anderson, 1993). At least two kinds of individual differences in judging causality have been identified. Firstly, the response criterion at which people report causal perceptions might differ (e.g., Beasley, 1968). Secondly, the stimulus parameters that contribute to causal perceptions might differ (e.g., Schlottmann and Anderson, 1993). Whereas in the first case the perceptual aspects might be similar across participants, in the latter case the judgments would be qualitatively different and might rely on different neural processes.

Questions of whether causality is perceived automatically, how to account for individual differences, and importantly, its neural instantiation remain unresolved. By parametrically varying spatial and temporal variables we wished to shed light on effects of spatial and temporal variables on the neural basis of the perception of causality. We tested the following alternate hypotheses. Firstly, if perceptual causality is automatic and universal, we predict minimal behavioral variability and consistent neural signatures across participants when perceiving causality. However, if perceptual causality is a variable experience, we predict behavioral differences and lack of neural consistency across individuals when perceiving causality.

\section{MATERIALS AND METHODS}

The stimuli were constructed using Strata-3D software. Each stimulus was $2 \mathrm{~s}$ long. The balls were shaded to give the impression of three dimensional objects and not flat discs. The first ball (colored blue) began to move $400 \mathrm{~ms}$ after animation onset and stopped moving after $1000 \mathrm{~ms}$. The blue ball always rolled along a horizontal plane and stopped once it came into contact with the second ball (colored red). The red ball varied in the direction and the time at which it began to move (see Figure 1). Speed and length of trajectory were the same for both balls in all conditions. All stimuli were rendered and converted to the Windows Media File format (WMV2/PAL; frame rate $=60$ frames per second, resolution $=720 \times 576$ pixels $)$.

\section{PARTICIPANTS}

Sixteen healthy participants took part in the study (nine male, seven female, mean age $25.9 \pm 3.6$ years, range $22-31$ years). All participants were right handed, native English speakers. All participants had normal or corrected-to-normal vision and none reported hearing deficits. One male participant's fMRI-data was excluded because of excessive movement during the fMRI procedure. All participants gave written informed consent prior to participation in the study. The University of Pennsylvania's Internal Review Board approved the study.

\section{EXPERIMENTAL DESIGN}

Our stimuli were animated video clips of a blue ball colliding with a red ball (a launching event). The stimuli varied in the direction of the subsequent movement of the red ball (seven angles: $0^{\circ}, 7.5^{\circ}, 15^{\circ}$, $22.5^{\circ}, 30^{\circ}, 45^{\circ}, 60^{\circ}$ with respect to the direction of movement of the blue ball) and the duration before the subsequent initial movement of the red ball (seven time delays: 0, 33, 67, 100, 133, 200, $267 \mathrm{~ms}$ ) after contact by the blue ball. All possible combinations of angle changes and time delays resulted in 49 different stimulus conditions (see Figure 1). Each of these 49 conditions was then flipped horizontally so that the balls were presented moving both left to right and right to left, resulting in a set of 98 stimuli. The videos were presented in random order and participants were instructed to judge the causal relationship between the balls (Did the blue ball cause the red ball to move? Yes or no). The responses were given with the right hand via two buttons of a non-magnetic response box [Index finger: Yes (causal); Middle finger: No (non-causal)]. Each video was followed by a fixation cross with a variable duration of $2000 \mathrm{~ms}$ to $8000 \mathrm{~ms}$ (average $5000 \mathrm{~ms}$ ).

\section{Behavioral pilot study}

We conducted a behavioral pilot experiment $(n=16)$ to determine the effectiveness of our manipulations when making judgments about causality. The results of the pilot experiment indicated that both spatial and temporal stimulus characteristics were used to judge causality. We found considerable variability between participants on the parameters used to make causality judgments, indicating that this task was suitable for further use in the fMRI study.

\section{MRI DATA ACQUISITION}

The video clips were presented via a mirror that reflected the computer screen. Participants wore ear plugs.

All MRI data were acquired on a Philips Achieva 3 T scanner. Functional images were acquired using a $\mathrm{T}_{2}{ }^{*}$ weighted echo planar image sequence $\left(\mathrm{TR}=3 \mathrm{~s}, \mathrm{TE}=30 \mathrm{~ms}\right.$, flip angle $90^{\circ}$, slice thickness $3 \mathrm{~mm}, 64 \times 64$ matrix, FoV $240 \mathrm{~mm}$, in-plane resolution $3.44 \mathrm{~mm} \times 3.44 \mathrm{~mm}, 50$ axial slices orientated parallel to the ACPC line covering the whole brain). Two runs of 240 volumes were acquired during the experiment. The onset of each trial sequence corresponded with scanner pulses.

\section{MRI DATA ANALYSIS}

SPM $^{1}$ standard procedures and templates were used to analyze fMRI data. After discarding the first two volumes to minimize T1saturation effects, all images were spatially and temporally realigned, normalized (resulting voxel size $4 \times 4 \times 4 \mathrm{~mm}^{3}$ ), smoothed ( $8 \mathrm{~mm}$ isotropic Gaussian filter) and high-pass filtered (cut-off period $128 \mathrm{~s})$.

${ }^{1}$ www.fil.ion.ucl.ac.uk 


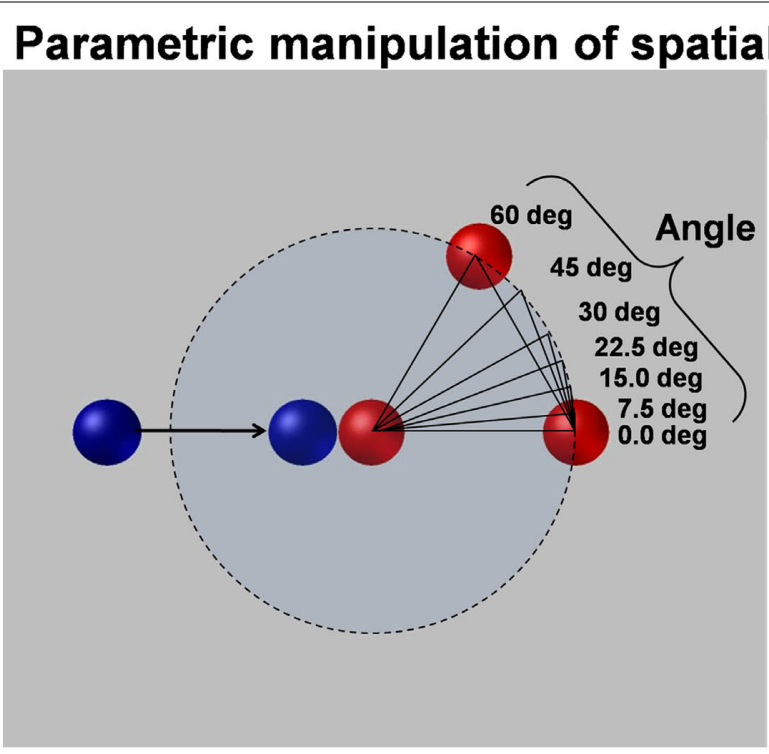

Task \& Stimulus presentation

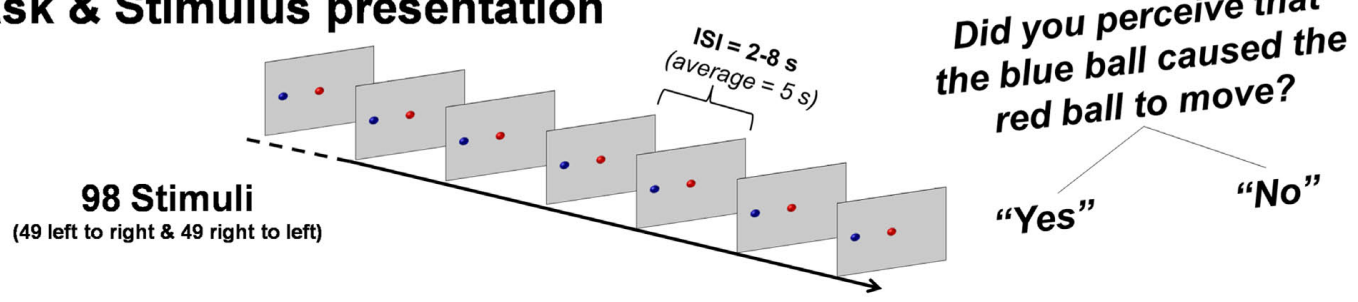

and temporal contiguity

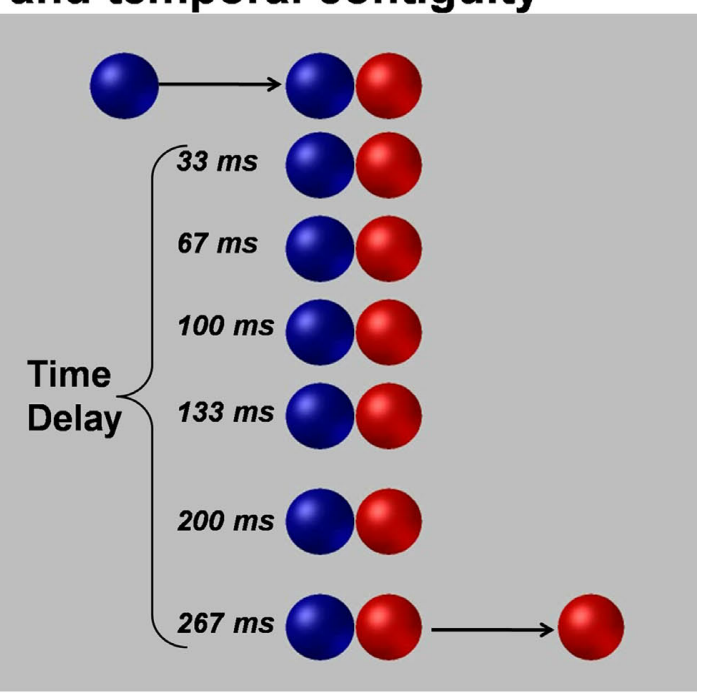
Did you perceive that the red ball to move?
Did you perceive that
FIGURE 1 | Parametric manipulation of spatial continuity and temporal contiguity. Illustrates the parametric violations of spatial continuity and temporal contiguity. All combinations of angle and time delay were presented to the subjects twice (once in which the balls moved left to right and once in which they moved right to left) in a pseudo-randomized order. For each trial the subject was instructed to press a button if they thought that the blue ball caused the red ball to move.
Statistical analyses were performed in a two-level, mixed-effects procedure. Two analyses were performed. The first analysis focused on the general differences in brain activation between trial judged as causal and trial judged as non-causal. Therefore, single-subject BOLD responses were modeled using a design matrix composed of the different onsets vectors of causal and non-causal judged events. Thus, on average 49 trials were in each condition (rage 15-83 trials; see behavioral results). To control for differences in stimulus characteristics and reaction times between causal and non-causal events three linear parameters were modeled as covariates of no interest including the corresponding stimulus parameters for each trial: angle, time delay and the corresponding reaction time of the judgment task. A second analysis focused on the response to stimulus characteristics independent of the causality judgment. Therefore, single-subject BOLD responses were modeled using just one onsetvector including all events $(n=98)$ regardless of the judgments of the participants. Thus, the stimulus parameters: angle, time delay and their interaction (angle $\times$ time delay) were correlated with the amplitude of the BOLD response. Parameter estimate ( $\beta$-) images for the HRF were calculated for each parameter and participant.

Random-effects group analyses were performed by entering contrast images into flexible-factorial analyses. Firstly, contrast images of causal and non-causal judged trials, secondly, images of angle, time delay and interaction from each participant were entered into a flexible-factorial analysis, respectively. A covariate (centered and in interaction with the conditions) was further created for the second analysis including the individual predictive values of angle, delay and their interaction for the judgment of causality. These regression parameters were calculated by logistic regression analyses ${ }^{2}$ (Hosmer and Lemeshow, 1989) predicting the judgment of causality for each participant individually by using the stimulus parameters angle, time delay and their interaction.

All reported activations are corrected for multiple comparisons at $p<0.05$ using the family wise error (FWE) correction implemented in SPM5. The reported voxel coordinates of activation peaks are located in MNI space. For anatomical localization, the functional data were referenced to probabilistic cytoarchitectonic maps (Eickhoff et al., 2007).

\section{RESULTS}

\section{BEHAVIORAL RESULTS}

Participants judged about half the events as causal ( mean $=51.03 \%$, SD 16\%; range 15.31-85.57\%). As expected, trials judged as causal had on average smaller angles and smaller time delays than non-causal trials $\left[\right.$ Angle: causal mean $=20.81^{\circ}, \mathrm{SD} 6.87^{\circ}$, non-causal mean $=31.53^{\circ}$,

${ }^{2}$ http://statpages.org/logistic.html 
SD 8.62, $t(15)=-3.27, p<0.01$; Time delay: causal mean $=66.87 \mathrm{~ms}$, SD $29.16 \mathrm{~ms}$, non-causal mean $=164.67 \mathrm{~ms}, \mathrm{SD} 27.23, t(15)=-7.81$, $p<0.001$; all two-tailed, $n=16$; see Figures 2 and 3 ]. The descriptive statistics for Angle must be evaluated in relation to the overall average angle of $25.71^{\circ}$. The descriptive statistics for time delay must be evaluated in relation to the overall average time delay of $114.29 \mathrm{~ms}$. Reaction times for non-causal judgments (mean $=1.91 \mathrm{~s}, \mathrm{SD}=0.18$ ) were slightly longer than those for causal judgments [ mean $=1.82$, $\mathrm{SD}=0.17$ s.; $t(15)=2.08, p<0.028$; one-tailed, $n=16]$.

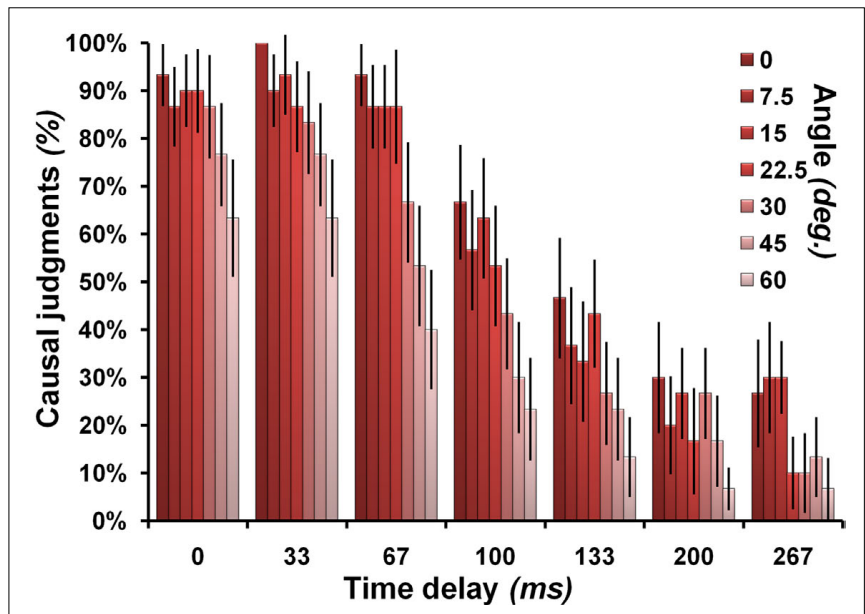

FIGURE 2 | Causal judgments in relation to stimulus characteristics. Illustrates the probability that a particular combination of angle and time delay was judged as causal. The angle manipulation is color-coded on a scale from dark red (no angle) to light red $\left(60^{\circ}\right)$. The error bars represent the standard error of the mean.
Logistic regression analyses ${ }^{3}$ (Hosmer and Lemeshow, 1989) predicting the judgment of causality for each participant individually by using the stimulus parameters angle, time delay and their interaction revealed large individual differences and no consistent effect of the interaction of angle and time delay on judging causality. Negative coefficients of the logistic regression parameters indicate that these subjects are sensitive to the specific parameter. Of the subjects $87.5 \%(n=14)$ were sensitive to time, $75 \%(n=12)$ to angle and $43.75 \%(n=7)$ to an interaction of the two. Viewed differently, $62.5 \%$ (10) of the subjects were sensitive to both time and angle violations, $25 \%$ (4) only to time and $12.5 \%$ (2) only to angle. A negative coefficient indicates that larger angles (or time delays) were associated with a lower probability that that stimulus was judged as causal.

To investigate the relationship between stimulus characteristics, RTs and causality judgments, individual parameters of each event were averaged across participants $(n=16)$ and correlated with each other. Correlation analyses revealed that stimulus parameters [Angle, Time delay and Interaction (Angle $\times$ Time)] were negatively correlated with the judgment of causality and positive correlated with reaction times (Angle $\times$ CJ: $r=-0.355, p<0.001$; Time delay $\times$ CJ: $r=-0.853, p<0.001$; Int. $\times$ CJ: $r=-0.720, p<0.001$; Angle $\times$ RT: $r=0.217, p=0.032$; Time delay $\times$ RT: $r=0.532$, $p<0.001$; Int. $\times$ RT: $r=0.426, p<0.001)$. Consequently RTs are negatively correlated with the judgment of causality $(\mathrm{RT} \times \mathrm{CJ}$ : $r=-0.561, p<0.001)$.

With regard to the logistic regression parameters we found a negative but not significant correlation between the parameters for angle and time delay $(r=-0.371, p=0.17)$.

${ }^{3}$ http://statpages.org/logistic.html

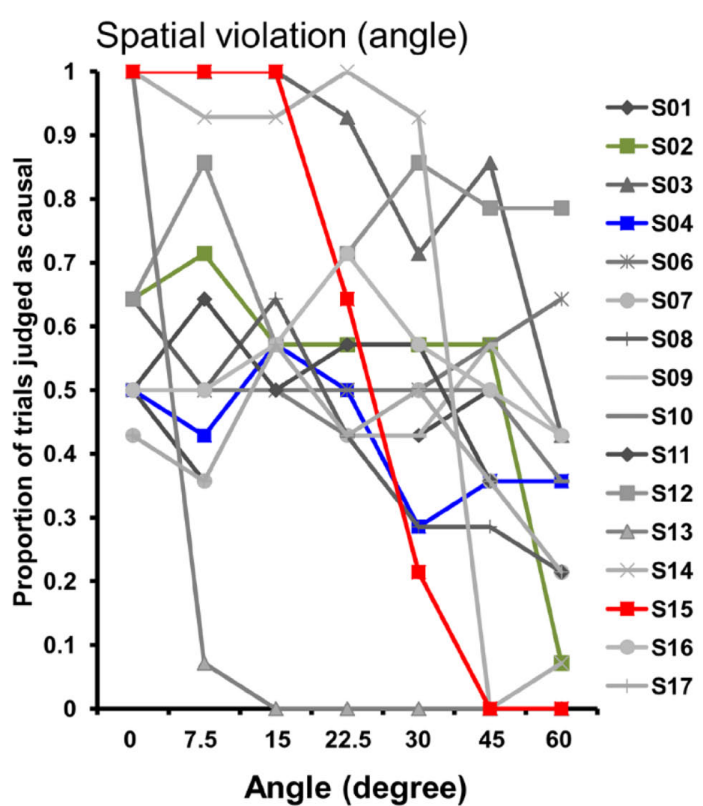

FIGURE 3 | Individual differences in causality judgments with regard to spatial and temporal violations. Illustrates the individual differences in the proportion of causality judgments separate for each subject and divided for angle and time manipulations. Each data-point represents the mean of all 14 trials

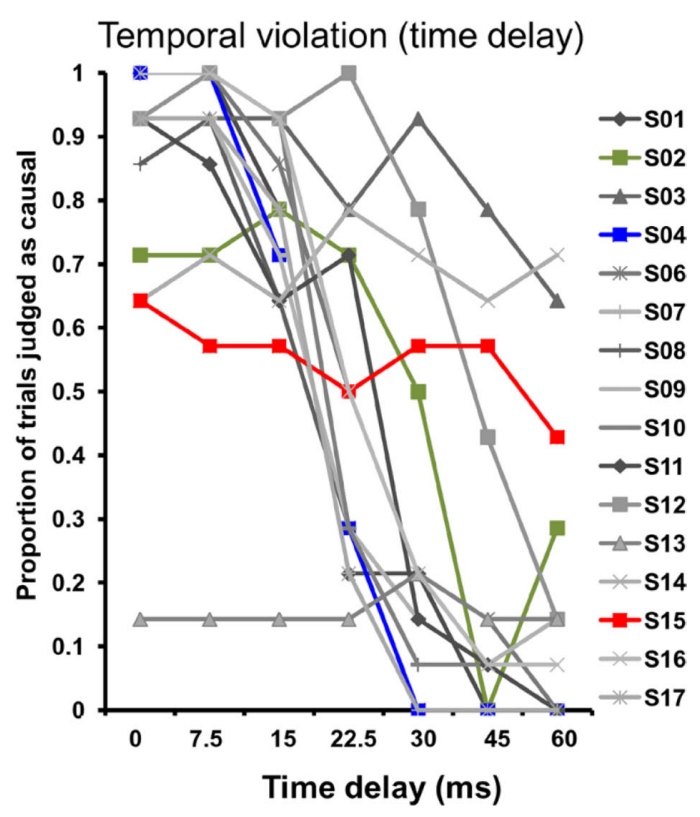

presented with the corresponding angle or time delay. Each line corresponds to a single subject. S02, S04 and S15 are highlighted to illustrate the different response pattern. These subjects are also shown in Figure 6 (SO4: blue, sensitive to time; S15: red, sensitive to angle; S02: green, sensitive to angle and time). 


\section{fMRI RESULTS}

All event trials regardless of whether they were judged as causal or not causal when contrasted to a fixation baseline activated a distributed areas of the brain including bilateral occipito-temporal and parietal brain regions, as well as precentral and cerebellar areas of the brain (see Figure 4 for illustration). The fMRI analysis contrasting the causal to non-causal judged trials did not reveal any consistent group activation patterns (at a significant threshold of $p<0.05$, FWE corrected). At a lower threshold ( $p<0.001,10$ voxels, uncorrected) we found only activation for non-causal in contrast to causal judged trials within the basal ganglia (left caudate nucleus: MNI $x, y, z=-12,12,12, t=4.97,33$ voxels; right caudate nucleus: MNI $x, y, z=16,16,-4, t=5.11,11$ voxels).

In contrast to the lack of consistent group-level activation patterns between causal and non-causal trials, individual differences in the sensitivity to spatial and/or temporal cues in judging cau- sality correlated with neural activity for the processing of spatial (increasing angle) and temporal (increasing time delay) stimulus characteristics. For this analysis we identified, for each participant individually, brain regions sensitive to parametric increase in spatial (angle from 0 to $60^{\circ}$ ) and temporal (time delay from 0 to $256 \mathrm{~ms}$ ) stimulus characteristics. Than we correlated the resulting activation maps with the individual predictive value of spatial and temporal characteristics for the judgment of causality (calculated with logistic regression analysis ${ }^{4}$.

Increasing sensitivity to angle for the judgment of causality correlated with increased activity predominantly in the right postcentral gyrus with cluster extensions across parts of the superior and inferior parietal cortex (and smaller clusters in more distributed

${ }^{4}$ Additionally we included the interaction term (angle $\times$ time delay) in all analysis. However, we found no significant effect of this variable.

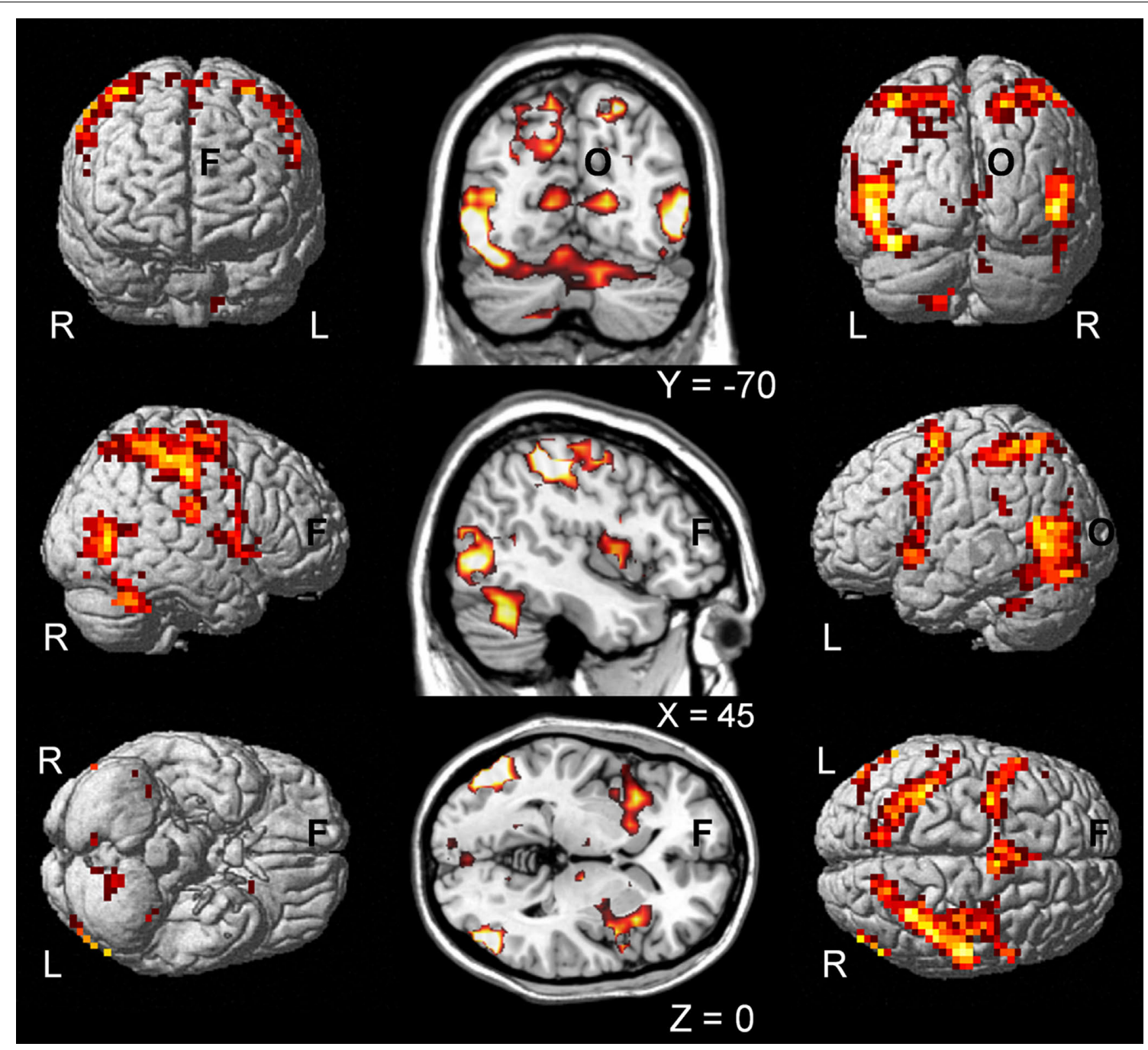

FIGURE 4 | Common activations for causal and non-causal trials. Shows the group activation map for common activation (based on a conjunction null analysis, SPM) of causal and non-causal judged trials in contrast to baseline (fixation cross) independent of increasing spatial and temporal violations and the individual judgment of causality. Activation maps are presented of single subjects render brain of SPM (left and right) and on coronal, sagittal and axial slices of a template brain (middle; MRIcro). L, left; R, right; F, frontal; O, occipital. 


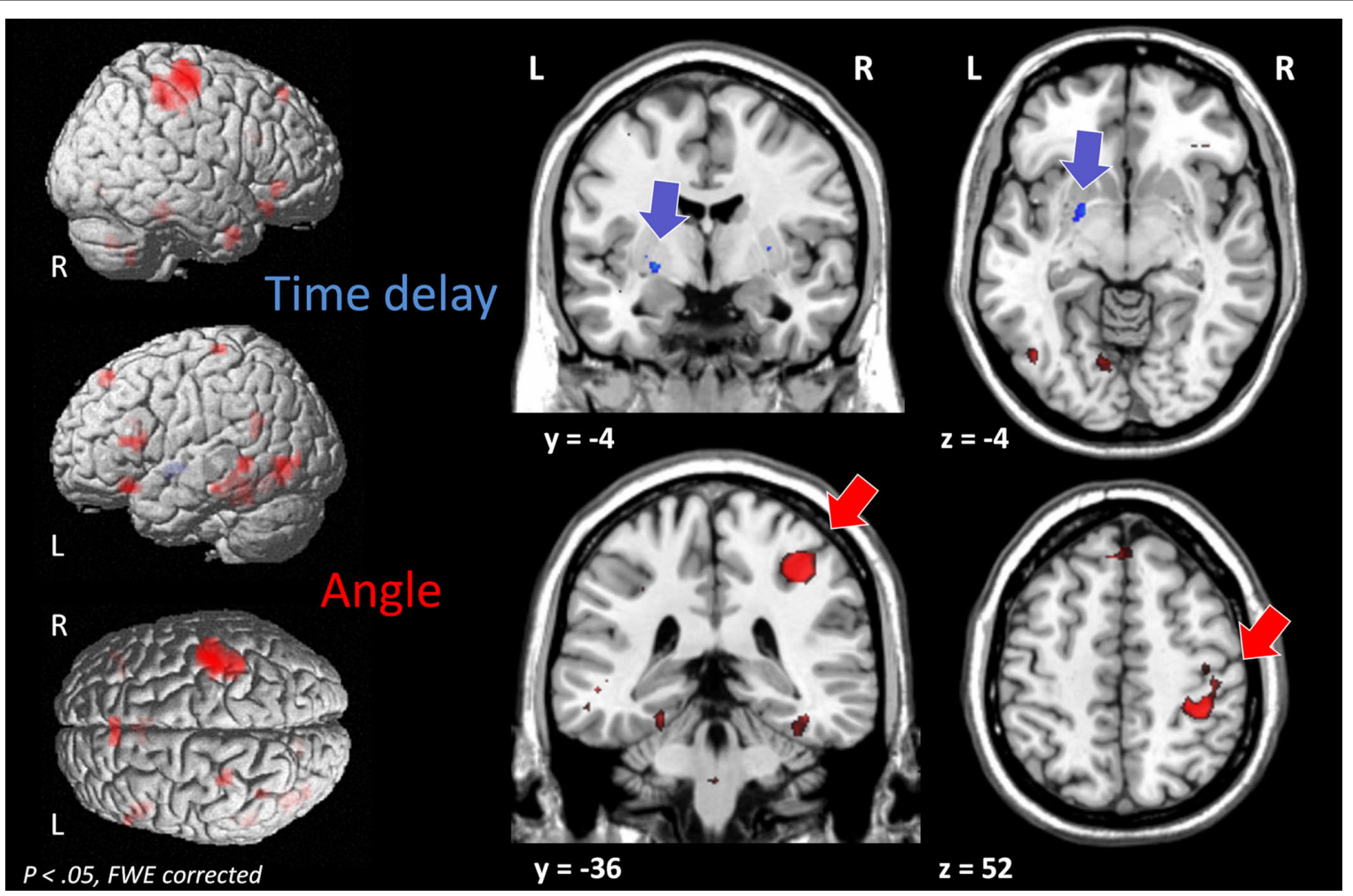

FIGURE 5 |The processing of spatial and temporal characteristics in relation to the judgments of causality. Shows the group activation map for the correlation of the processing of stimulus characteristics their individual predictive value for the judgment of causality. Activation maps for increasing angle and time delay for each subject were correlated at the group level with the predictive values of the logistic regression analyses for angle (red) and time delay (blue), respectively. brain regions) (Figure 5). By contrast, increasing sensitivity to time delay for the judgment of causality correlated with increased activity in the basal ganglia (left putamen) (see Figure 5). Importantly, as illustrated in Figure 6, these results are based on correlations of inter-individual differences in brain activation in response to spatial and temporal stimulus characteristics and inter-individual differences in the stimulus parameters used for the judgment of causality. (For complete activation patterns of both contrasts, see Table 1).

For general effects of temporal and spatial violations at a group level we found three significant activation patterns that differed from the analyses including the individual predictive values for the judgments of causality. The group average of the parametric analysis of increase in angle and time delay revealed three areas not related to the judgment of causality (Angle: left temporal pole, MNI $x, y, z=-40$, $12,-32, t=6.74,5$ voxels; Time delay: left occipito-temporal junction: MNI $x, y, z=-36,-56,-16, t=6.52,10$ voxels; Interaction: left temporal pole, MNI $x, y, z=-40,16,-32, t=5.95,3$ voxels). These differences are not surprising considering the high individual variability between subjects in these contrasts (see Figure 6).

Violations of temporal contiguity and spatial continuity led to longer reaction times and a reduction in the proportion of events judged as causal. Violations of temporal contiguity were associated with increased activity in the left putamen and violations of spatial continuity were correlated with more distributed activity with the highest activation in right postcentral gyrus with cluster extensions across parts of the superior and inferior parietal cortex (see Table 1). Individual differences in activation of these areas correlated with how one judged the causal relationship of the stimuli. We found no common activations and no significant effects for the interaction of angle and time delay.

\section{DISCUSSION}

Humans are adept at inferring causal relationships in everyday life. This fundamental feature of human cognition allows us to theorize about and predict future states of the world. However, mechanisms underlying even the simple perception that the impact of one ball causes another to move are not well understood. Our study investigated the neural basis of the perception of causality with attention to individual differences in how spatial and temporal characteristics predict causality judgments.

We found bilateral occipito-parietal and frontal brain activation in response to ball movements, which is consistent with previous literature about movement perception (e.g., Billino et al., 2009), the perception of launching events (Blakemore et al., 2001) and extraction of specific movement parameters in event perception (e.g., Zacks et al., 2001, 2006; Zacks, 2004). However, we did not find consistent brain activation differences between events judged as causal and those judged as non-causal. Thus, it is unlikely that universal causality related structures are engaged by the perception of causality. However, we did find that individual differences in judging causality are associated with distinct neural responses. Participants differed in their sensitivity to spatial and temporal characteristics 


\section{Individual subjects' activation for increasing time gap (blue) and angle (red)}
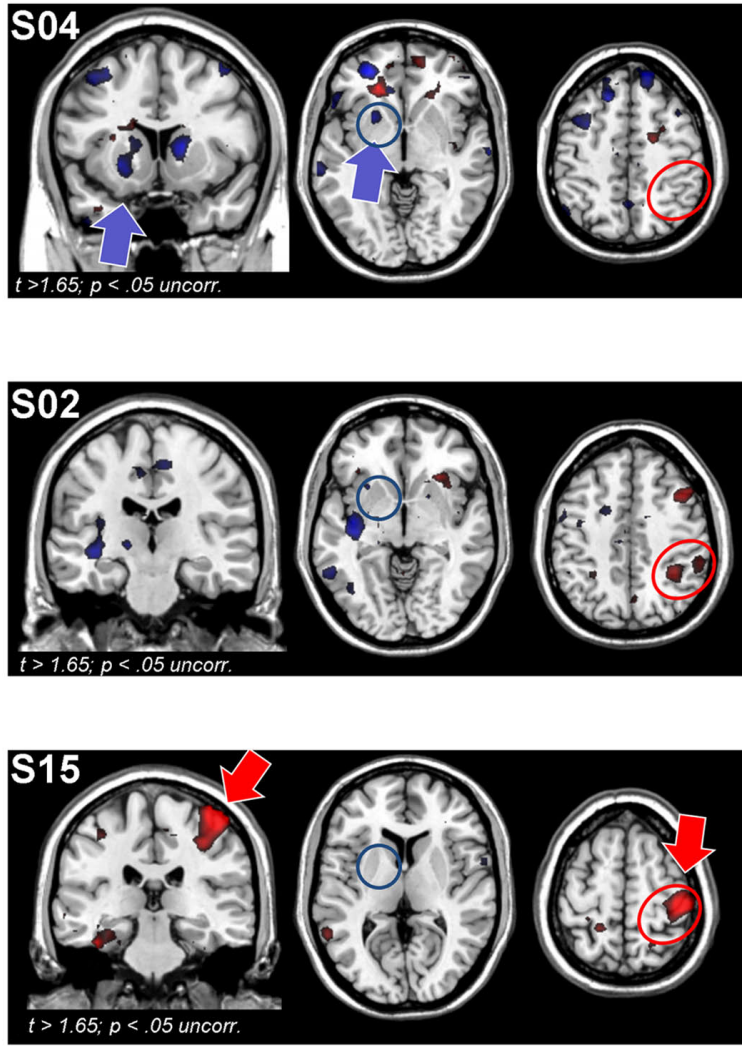

FIGURE 6 | Brain activation and causality judgments of three individual participants. Illustration of three individual participants with different patterns of brain activation in response to increasing angle (red) and time delay (blue; left). These participants also show distinct response patterns in their judgments of causality (center) and corresponding predictive values of
Ind. Response Pattern

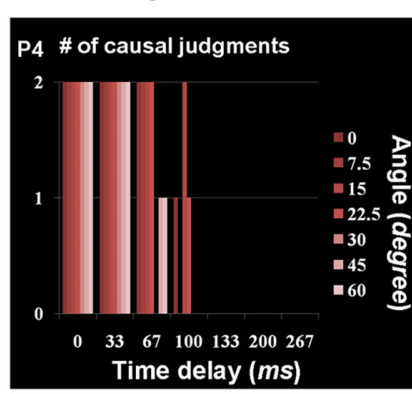

Ind. Logistic Reg. parameter

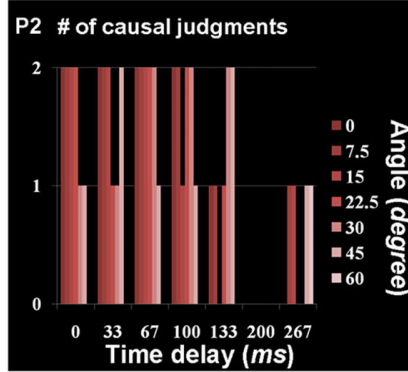

P15 \# of causal judgments

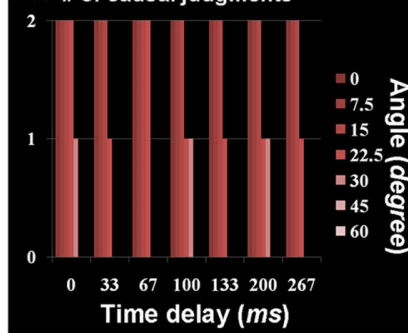

Variable

Angle

Coeff.

$-0.4471$

$-0.3773$

Time gap $\quad-0.3773$

$\begin{array}{lr}\text { Ang x Time } & \mathbf{0 . 0 0 7 8} \\ \text { Intercept } & \mathbf{2 1 . 8 2 8 4}\end{array}$ of events when perceiving causal relationships. Individuals' sensitivity to spatial information when perceiving causality correlated with activation of the right parietal lobe, an area often implicated in spatial perception (Shulman et al., 1999; Keehner et al., 2006). Attention to path trajectories engage the dorsal visual stream ( Wu et al., 2008) and are likely responsible for detecting violations of spatial continuity. By contrast, individuals' sensitivity to time information when judging causality correlated with increased activation within the left basal ganglia. The basal ganglia are implicated in the perception of time delays in sub- and supra-second range (see Lewis and Miall, 2003; Meck et al., 2008 for a review). Our results reveal that individual differences in perceptual causality are accompanied by different neural signatures depending on whether individuals are sensitive to time or to space. Time and space seem to be elemental constituents that contribute to causal perceptions.

In contrast to the focal activation of the left putamen for time delays predicting the judgment of causality, we found a more distributed brain network for the spatial domain. In addition to the right parietal lobe, the lateral and medial frontal and occipital angle and delay from the logistic regression analyses (right). Participants with more activation in the basal ganglia are more likely to attend to time delays, whereas participants with more activity in the right parietal lobe were more sensitive to spatial violations (see Figure $\mathbf{5}$ for group analysis) and temporal brain regions were activated if differences in trajectory between the balls were used to judge causality. The occipitotemporal activations may reflect enhanced visual attention, which is possibly mediated by the frontal lobe (bilateral IFG, medial frontal regions; e.g., Banich et al., 2000; Thomsen et al., 2005; Buschman and Miller, 2007; Natale et al., 2009). The fact that we did not find a similarly extensive network for the temporal domain may be an effect of the thresholds being used in the analysis. For example, at a higher threshold (e.g., $t>8$ ) for the spatial contrast only the parietal lobe activity remains.

\section{PREVIOUS STUDIES}

Only a few studies have examined the neural bases for causal perception. These studies have focused on increases in activation during conditions defined as causal than in those defined as noncausal (Blakemore et al., 2001; Fugelsang et al., 2005). They find occipital and parietal region activations (Blakemore et al., 2001) and right hemisphere (Fugelsang et al., 2005) when perceiving causal relationships. Fugelsang et al. (2005) further distinguished 
Table 1 |Activations related to logistic regression parameters.

\begin{tabular}{|c|c|c|c|c|c|c|}
\hline \multirow[t]{2}{*}{ Anatomical region } & \multirow[t]{2}{*}{ Cluster extend } & \multicolumn{3}{|c|}{ Coordinates } & \multirow[t]{2}{*}{ No. of voxels } & \multirow[t]{2}{*}{$t$-value } \\
\hline & & $x$ & $y$ & $z$ & & \\
\hline Postcentral gyrus (R) & $\mathrm{BA} 1,2,3,4,6,7 ; \mathrm{SPL} ; \mathrm{IPC}$ & 36 & -36 & 52 & 84 & 9.15 \\
\hline Fusiform gyrus (L) & & -32 & -44 & -16 & 23 & 7.44 \\
\hline Cerebellum (L) & $\mathrm{BA} 17,18 ; \mathrm{V} 3, \mathrm{~V} 4$ & -8 & -72 & -4 & 14 & 7.43 \\
\hline Inferior frontal gyrus (L) & p. Orbitalis & -28 & 24 & -16 & 12 & 7.08 \\
\hline Inferior temporal gyrus (R) & Hipp. (EC) & 36 & 4 & -36 & 7 & 7.07 \\
\hline Inferior frontal gyrus (L) & BA 44,45 & -48 & 20 & 12 & 8 & 6.92 \\
\hline Middle temporal gyrus (L) & ITG & -56 & -44 & 0 & 13 & 6.82 \\
\hline Fusiform gyrus (R) & & 40 & -36 & -16 & 5 & 6.78 \\
\hline Superior medial gyrus (L) & & -4 & 36 & 52 & 9 & 6.69 \\
\hline Anterior cingulate cortex $(\mathrm{L})$ & & -8 & 20 & 20 & 6 & 6.18 \\
\hline Middle temporal gyrus (L) & & -40 & -52 & 20 & 5 & 6.15 \\
\hline Cerebellum (R) & & 24 & -56 & -48 & 3 & 6.00 \\
\hline Middle orbital gyrus (R) & & 28 & 28 & -16 & 4 & 5.71 \\
\hline Precentral gyrus (L) & BA 6,4 & -32 & -28 & 68 & 3 & 5.51 \\
\hline \multicolumn{7}{|c|}{ ACTIVATION FOR INCREASING TIME DELAY IN RELATIONTOTHE PREDICTIVE VALUE OFTIME FOR THE JUDGMENT OF CAUSALITY } \\
\hline Putamen (L) & & -24 & -4 & -4 & 4 & 6.04 \\
\hline
\end{tabular}

Brain activation for increasing angle and time delay correlated with parameters of the logistic regression analyses (angle and time delay), respectively. Anatomical regions, cluster extent, corresponding coordinates, and significance of the peak voxels (t-value) are shown for clusters with $p<0.05$ corrected for multiple comparisons (FWE).BA, Brodmann area; L, left hemisphere; $R$, right hemisphere; Hipp, hippocampus; ITG, inferior temporal gyrus; IPC, inferior parietal cortex; SPL, superior parietal lobule.

both common (right prefrontal) and unique (right parietal and right temporal) regions of activation as a function of spatial and temporal processing of contiguity, respectively. Medial frontal activations (Fonlupt, 2003) and left hemispheric processes have also been reported to reflect inferential rather than perceptual processes in judging causality (Roser et al., 2005).

The discrepancies between our and previous findings might be due to differences of the stimulus manipulation (parametric violations vs. condition comparison applied by Blakemore et al., 2001; Fonlupt, 2003; Fugelsang et al., 2005), the experimental design (event related vs. block design applied by Fugelsang et al., 2005) and the insensitivity to individual differences in previous studies. Importantly our design, unlike previous studies, allowed us to identify stimulus driven brain responses in relation to individual differences in the perception of causality. Furthermore, in past research, most spatial violations were defined as physical distance between stimuli (the objects never make contact) (e.g., Fugelsang et al., 2005; Roser et al., 2005; Schlottmann et al., 2006), rather than changes in trajectory as presented here.

Our data reveal that the perception of causality is not simply a function of the occipito-parietal network (Blakemore et al., 2001) or the right hemisphere (Fugelsang et al., 2005; Roser et al., 2005). Rather, the neural instantiation of causality itself varies, depending on individual sensitivities to spatial and temporal characteristics of events.

\section{NATURAL EXPECTATION}

Violations of spatial continuity and temporal contiguity produced increase in reaction times and greater activation of specific brain regions. Spatial continuity and temporal contiguity violations appear to be unexpected resulting in longer processing time. Thus, causal perception may be the default mode when viewing two moving objects interacting. Past research on explicit timing (see Coull and Nobre, 2008) point to the role of the basal ganglia. Similarly spatial cues about movement direction produce larger modulations of brain activation than neutral cues (with respect to a passive viewing baseline) in the anterior and posterior intraparietal sulcus (Shulman et al., 1999).

A wider angle is more salient and less expected in subjects sensitive to angle information. Thus, both attention and the processing of movement trajectory (maybe as a result of the attention effect) are likely to be enhanced for wider angles in these subjects. By contrast, attention and processing of angle information will be unaffected by wider angles in participants only sensitive to time delays, and vice versa. In this study we cannot completely disentangle attention processes from spatial and temporal processing mechanisms. However, the distinct activation pattern between "Temporal-Participants" (basal ganglia) and "Spatial-Participants" (parietal lobe) suggest at least that distinct domain specific processes are engaged, which could include domain specific attention effects. 
Schlottmann and Anderson (1993) showed differences in the kind of stimulus parameters that contribute to individual judgments of causality. They believed that the selection of a stimulus parameter for the rating of causality is a cognitive rather than a perceptual process. If this assumption is true, such a cognitive process might be correlated with brain activation independent of the perceptual parameters of the event. Certainly, top-down processes can modulate specific perceptual processes (e.g., Buschman and Miller, 2007). However, such top-down processes would be expected to produce pattern of consistent activation independent of stimulus characteristics most likely within the prefrontal cortex. By contrast, if the evaluation is perceptually driven, then one would predict patterns of activation within perceptual brain regions correlating with causal judgments causality. Our data support the latter possibility. Different sensitivities in spatial and temporal perceptual systems account for individual differences when judging cause and effect relationships.

\section{CONCLUSION}

Debates about the nature of the perception of causality have spanned decades and are receiving increasing attention (see

\section{REFERENCES}

Banich, M. T., Milham, M. P., Atchley, R., Cohen, N. J., Webb, A., Wszalek, T., Kramer, A. F., Liang, Z. P., Wright, A., Shenker, J., and Magin, R. (2000). fMri studies of Stroop tasks reveal unique roles of anterior and posterior brain systems in attentional selection. J. Cogn. Neurosci. 12, 988-1000.

Beasley, N. A. (1968). The extent of individual differences in the perception of causality. Can. J. Psychol. 22, 399-407.

Billino, J., Braun, D. I., Böhm, K.-D., Bremmer, F., and Gegenfurtner, K. R. (2009). Cortical networks for motion processing: effects of focal brain lesions on perception of different motion types. Neuropsychologia 47, 2133-2144.

Blakemore, S. J., Boyer, P., Pachot-Clouard, M., Meltzoff, A., Segebarth, C., and Decety, J. (2003). The detection of contingency and animacy from simple animations in the human brain. Cereb. Cortex 13, 837-844.

Blakemore, S. J., Fonlupt, P., PachotClouard, M., Darmon, C., Boyer, P., Meltzoff, A. N., Segebarth, C., and Decety, J. (2001). How the brain perceives causality: an event-related fMRI study. Neuroreport 12, 3741-3746.

Buschman, T. J., and Miller, E. K. (2007). Top-down versus bottom-up control of attention in the prefrontal and posterior parietal cortices. Science 315, 1860-1862.

Coull, J., and Nobre, A. C. (2008). Dissociating explicit timing from temporal expectation with fMRI. Curr. Opin. Neurobiol. 18, 137-144.
Eickhoff, S. B., Paus, T., Caspers, S., Grosbras, M. H., Evans, A. C., Zilles, K., and Amunts, K. (2007). Assignment of functional activations to probabilistic cytoarchitectonic areas revisited. Neuroimage 36, 511-521.

Fonlupt, P. (2003). Perception and judgement of physical causality involve different brain structures. Brain Res. Cogn. Brain Res. 17, 248-254.

Fugelsang, J. A., Roser, M. E., Corballis, P. M., Gazzaniga, M. S., and Dunbar, K. N. (2005).Brain mechanisms underlying perceptual causality. Brain Res. Cogn. Brain Res. 24, 41-47.

Gemelli, A., and Cappellini, A. (1958). The influence of the subject's attitude in perception. Acta Psychol. 14, 12-23.

Gruber, H. E., Fink, C. D., and Damm, V. (1957). Effects of experience on the perception of causality. J. Exp. Psychol. 53, 89-93.

Guski, R., and Troje, N. F. (2003). Audiovisual phenomenal causality. Percept. Psychophys. 65,789-800.

Hosmer, D. W., and Lemeshow, S. (1989). Applied Logistic Regression. New York: Wiley.

Hume, D. (1740/1960). A Treatise of Human Nature. Oxford: Clarendon.

Hume, D. (1748/1977). An Enquiry Concerning Human Understanding. Indianapolis: Hackett.

Keehner, M. Guerin, S. Miller, M. Turk, D., and Hegarty, M. (2006). Modulation of neural activity by angle of rotation during imagined spatial transformations. Neuroimage 33, 391-398.

Wagemans et al., 2006). Our results present a combination of two hypotheses under consideration. Consistent with the view that causality can be perceived and is a universal human predisposition, our participants were biased to infer causality when two objects make contact and move. However, we did not find evidence of a universal instantiation of perceptual causality in the brain. Consistent with the view that the experience of causality varies across individuals, we found that perceptual causality is built upon more elemental sensitivities to spatial continuities and temporal contiguities. Furthermore, these sensitivities vary across individuals. These elemental spatial and temporal sensitivities are instantiated in distinct neural networks, which in turn trigger the perception that movement of one object causes the movement of another.

\section{ACKNOWLEDGMENTS}

We thank L. Morgan for help with stimuli preparation and G. Aguirre for suggestions on the fMRI-data analyses. This work was supported by NIH RO1 HD050199 and RO1 DC008779 and a subcontract under NSF SBE0541957.

Leslie, A. M. (1982). The perception of causality in infants. Perception 11, 173-186.

Leslie,A.M., and Keeble, S. (1987). Do sixmonth-old infants perceive causality? Cognition 25, 265-288.

Lewis, P. A., and Miall, R. C. (2003) Distinct systems for automatic and cognitively controlled time measurement: evidence from neuroimaging. Curr. Opin. Neurobiol. 13 250-255.

Meck, W.H., Penney, T. B., and Pouthas, V. (2008). Cortico-striatal representation of time in animals and humans. Curr. Opin. Neurobiol. 18, 145-152.

Michotte,A.E.(1946/1963). The Perception of Causality (Translated by T. R. Miles and E. Miles). London: Methuen (Original published in 1946).

Natale, E., Marzi, C. A., and Macaluso, E. (2009). FMRI correlates of visuospatial reorienting investigated with an attention shifting double-cue paradigm. Hum. Brain Mapp. 30, 2367-2381.

Oakes, L. M., and Cohen, L. B. (1990). Infant perception of a causal event. Cogn. Dev. 5, 193-207.

Powesland, P. F. (1959). The effect of practice upon the perception of causality. Can. J. Psychol. 13, 155-168.

Roser, M. E., Fugelsang, J. A., Dunbar, K. N., Corballis, P.M., and Gazzaniga, M S. (2005). Dissociating processes supporting causal perception and causal inference in the brain. Neuropsychology 19, 591-602.

Schlottmann, A., and Anderson, N. H. (1993). An information integration approach to phenomenal causality. Mem. Cogn. 21, 785-801.

Schlottmann, A., Ray, E., Mitchell, A., and Demetriou, N. (2006). Perceived physical and social causality in animated motions: spontaneous reports and ratings. Acta Psychol. 123, 112-143.

Schlottmann, A., and Shanks, D. R. (1992). Evidence for a distinction between judged and perceived causality. Q. J. Exp. Psychol. A 44, 321-342.

Scholl, B. J., and Tremoulet, P. (2000). Perceptual causality and animacy. Trends Cogn. Sci. 4, 299-309.

Shulman, G. L., Ollinger, J.M.,Akbudak, E., Conturo, T. E., Snyder, A. Z., Petersen, S. E., and Corbetta, M. (1999). Areas involved in encoding and applying directional expectations to moving objects. J. Neurosci. 19, 9480-9496.

Thomsen, T., Specht, K., Ersland, L., and Hugdahl, K. (2005). Processing of conflicting cues in an attention-shift paradigm studied with $\mathrm{fMRI}$. Neurosci. Lett. 380, 138-142.

Wagemans, J., van Lier, R., and Scholl, B. J. (2006). Michotte's heritage in perception and cognition research. Acta. Psychol. 123, 1-19.

Wolpert, L. (2006). Six Impossible Things Before Breakfast. London: Faber \& Faber.

Wolpert, L. (2009). Cognition: evolution does help to explain how minds work. Nature 459, 506.

Wu, D., Morganti, A., and Chatterjee, A. (2008). Neural substrates of processing path and manner information of 
a moving event. Neuropsychologia 46, 704-713.

Zacks, J. M. (2004). Using movement and intentions to understand simple events. Cogn. Sci. 28, 979-1008.

Zacks, J. M., Braver, T. S., Sheridan, M. A., Donaldson, D. I., Snyder, A. Z., Ollinger, J. M., Buckner, R. L., and Raichle, M. E. (2001). Human brain activity time-locked to perceptual event boundaries. Nat. Neurosci. 4 , 651-655.

Zacks, J. M., Swallow, K. M., Vettel, J. M., and McAvoy, M. P. (2006). Visual motion and the neural correlates of event perception. Brain Res. 1076, 150-162.

Conflict of Interest Statement: The authors declare that the research was con- ducted in the absence of any commercial or financial relationships that could be construed as a potential conflict of interest.

Received: 05 February 2010; paper pending published: 25 February 2010; accepted: 17 March 2010; published online: 06 April 2010.

Citation: Straube B and Chatterjee A (2010) Space and time in perceptual cau- sality. Front. Hum. Neurosci. 4:28. doi: 10.3389/fnhum.2010.00028

Copyright $(0) 2010$ Straube and Chatterjee. This is an open-access article subject to an exclusive license agreement between the authors and the Frontiers Research Foundation, which permits unrestricted use, distribution, and reproduction in any medium, provided the original authors and source are credited. 